\title{
Cutaneous lichen amyloidosis
}

National Cancer Institute ( $\mathrm{NCl})$

\section{Source}

National Cancer Institute (NCI). Cutaneous lichen amyloidosis.

Itchy papules that are brownish-red in color and typically appear on the shins, thighs, feet, or neck. 